\title{
Plasma-Charging Damage of Floating MIM Capacitors
}

\author{
Zhichun Wang, Member, IEEE, Jan Ackaert, Cora Salm, Member, IEEE, Fred G. Kuper, Member, IEEE, \\ Marnix Tack, Member, IEEE, Eddy De Backer, Member, IEEE, Peter Coppens, Luc De Schepper, and Basil Vlachakis
}

\begin{abstract}
In this paper, the mechanism of plasma-charging damage (PCD) of metal-insulator-metal (MIM) capacitors as well as possible protection schemes are discussed. A range of test structures with different antennas simulating interconnect layout variations have been used to investigate the mechanism of PCD of MIM capacitors. Based on the experimental results, two models are presented, describing the relation between the damage and the ratio of the area of the exposed antennas connected to the MIM capacitors plates. New design rules are proposed in order to predict and automatically flag possible PCD sites. Furthermore, layout solutions to reduce PCD are suggested.
\end{abstract}

Index Terms-Antenna ratio (AR), metal-insulator-metal (MIM), plasma-charging damage (PCD).

\section{INTRODUCTION}

$\mathbf{H}$ IGH-PERFORMANCE mixed-signal and RF circuits built in CMOS or BiCMOS require integrated capacitors with high voltage independency, high quality factor, good capacitor matching, precision control of capacitor values and low parasitic capacitance, along with high reliability and a low defect density. Conventional high-density capacitors such as poly-substrate [1], poly-poly [2] and metal-poly [3], [4] structures suffer from low voltage independency due to voltage induced depletion effects as well as a large parasitic capacitance due to their proximity to the substrate.

The metal-insulator-metal (MIM) capacitors used for this study have low parasitic capacitance, especially when fabricated on metal 2 or higher, as well as a high quality factor $(Q)$ for RF circuit applications [5]. In the paper presented here, high-density MIM capacitors with capacitance densities of 1.0 to 2.0 $\mathrm{fF} / \mu \mathrm{m}^{2}$ and silicon nitride dielectrics deposited by plasma-enhanced chemical vapor deposition (PECVD), have been integrated into the backend metallization layers of a CMOS and a BiCMOS process flow. The capacitor breakdown voltage, linearity and reliability meet mixed-signal circuit requirements. A defect density less than 0.5 defects $/ \mathrm{cm}^{2}$ has been achieved for the test structures with $2.0 \mathrm{fF} / \mu \mathrm{m}^{2}$ specific capacitance. A quality factor $Q>10$ at frequencies of 1 and $2 \mathrm{GHz}$ was measured indicating its usefulness for RF applications [6].

Manuscript received August 13, 2003; revised March 18, 2004. The review of this paper was arranged by Editor J. Vasi.

Z. Wang and F. G. Kuper are with the MESA+ Research Institute/University of Twente, Enschede $7500 \mathrm{AE}$, The Netherlands and also with Philips Semiconductors, Nijmegen, The Netherlands (e-mail: Z.Wang@utwente.nl).

J. Ackaert, M. Tack, E. De Backer, P. Coppens, L. De Schepper, and B. Vlachakis are with AMI Semiconductor, Oudenaarde B-9700, Belgium.

C. Salm is with the MESA+ Research Institute/University of Twente, Enschede 7500 AE, The Netherlands.

Digital Object Identifier 10.1109/TED.2004.829518
The dielectric in MIM capacitors is much thicker than the MOS gate oxide-thickness and the antenna ratio (AR) $\left[\mathrm{AR}_{(\mathrm{T} / \mathrm{D})}\right]$ defined as the area ratio of top antenna versus dielectric is typically low and even near unity. Because of the small ARs, one does not expect these capacitors to suffer from plasma-charging damage (PCD) as rapidly as with thin gate oxides. However, previous studies found thicker oxides to be very sensitive to PCD. It was argued that thicker oxides require a higher voltage but less current to break down [7]. Harris stated that MIM capacitors can be damaged only when the bottom plate is grounded and the top plate is connected to a large conductor (antenna) that is exposed to a plasma [8].

In this paper however, we will demonstrate that floating MIM capacitors, without any substrate contact are also very sensitive to PCD. The interconnect lines connected to the top plate is referred to as top antenna, and the interconnect lines connected to bottom plate is referred to as bottom antenna in this paper. The $\mathrm{AR}_{(\mathrm{T} / \mathrm{B})}$, area ratio of top antenna versus bottom antenna, is a more critical parameter for floating MIM capacitor than $\mathrm{AR}_{(\mathrm{T} / \mathrm{D})}$, which is commonly used in studying PCD in the gate oxide of MOS transistors. Severe PCD occurs if the interconnect lines connected to the two MIM capacitor plates have a large difference in area, though the $\mathrm{AR}_{(\mathrm{T} / \mathrm{D})}$ is low and even near unity. The damage becomes larger with the increase of $\mathrm{AR}_{(\mathrm{T} / \mathrm{B})}$, independent of which plate is connected to the largest antenna. The mechanism and the protection schemes of PCD of floating MIM capacitors, are discussed here. Additional design rules are proposed, in order to predict and automatically flag the possible charge-damage. Furthermore, layout solutions to reduce the damage are suggested.

\section{EXPERIMENTAL}

\section{A. MIM Capacitor Processing}

The MIM capacitor is formed using a metal 1 layer as bottom plate, 20 to $60 \mathrm{~nm}$ silicon nitride as insulator, and an additional thin-metal 1.5 layer as top plate. The top plate is connected to metal 2 by vias. Figs. 1 and 2 show a schematic and a scanning electron microscopy (SEM) cross section of the MIM capacitor built on metal 1. A short description of the process flow follows. First, the bottom plate is formed by a standard metal 1 deposition of a Ti-TiN-AlCu-TiN stack. The thin silicon nitride film is deposited by PECVD followed by the deposition of the top metal plate (30-nm TiN, 200-nm AlCu, 30-nm TiN). Then, the top plate layer is patterned and etched with a selective etch chemistry with etch stop on the insulator film. Subsequently, the insulator film together with the bottom plate are patterned 


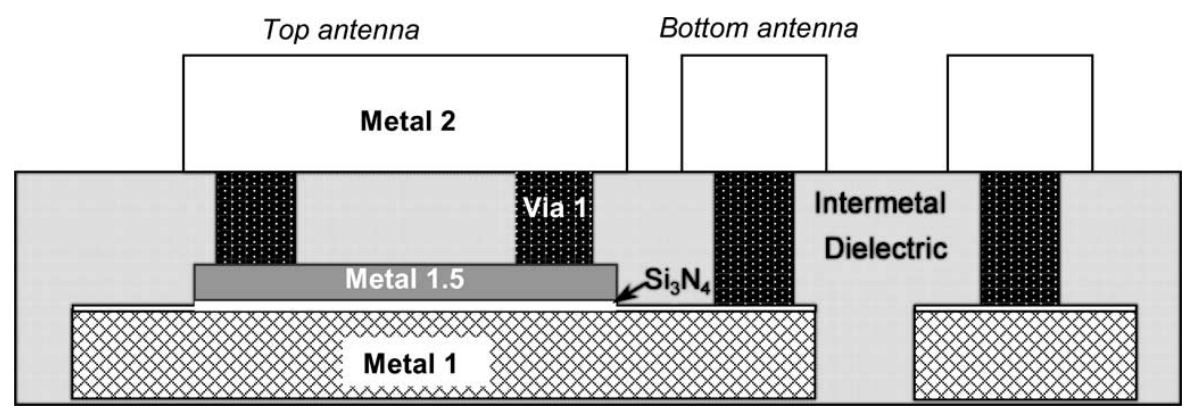

Fig. 1. Schematic diagram of MIM capacitor integrated on metal 2 of a backend CMOS.

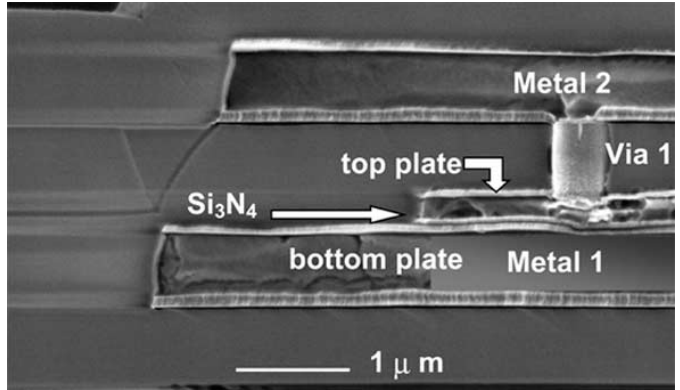

Fig. 2. Cross section SEM picture across a typical MIM capacitor. Note that the shallow via 2 provides the connection between the top plate of the capacitor to metal 3 . Connection of bottom plate (metal 2) to metal 3 is provided by a deep via 2 not depicted here.

and etched in sequence. The process flow continues with the standard CMOS backend flow sequence of intermetal dielectric (IMD) deposition, via 1 formation, and metal 2 patterning; followed by IMD deposition on metal 2, via 2 formation, and metal 3 patterning; finally finished with passivation. The IMD depositions consist of an $\mathrm{SiO}_{2}$ layer deposited by a medium density PECVD tool at $400{ }^{\circ} \mathrm{C}$. In order to improve the particle performance of the process a plasma power ramp-down (PPRD) step is added at the end of deposition process. This step prevents the deposition on the chamber walls releasing and affecting the wafer surface.

The MIM capacitor is implemented using the above scheme in a CMOS backend flow with 1.1- $\mu \mathrm{m}$ metal pitch design rules [9].

\section{B. Test Structures}

More than 400 systematically arranged test structures have been designed and processed in order to investigate and characterize the effect of charging during processing on the yield and reliability of the MIM capacitors. MIM capacitors have been designed with an area of $5 \times 5 \mu \mathrm{m}^{2}, 20 \times 20 \mu \mathrm{m}^{2}$ and $20 \times 5 \times 5 \mu \mathrm{m}^{2}$ in parallel. Antennas with different features are connected to top and bottom plate, in order to simulate the interconnect layout design of MIM capacitors. The area of the antennas is designed in a range from $20 \mu \mathrm{m}^{2}$ up to $120000 \mu \mathrm{m}^{2}$ or consists only of a small connection pad $\left(1.36 \mu \mathrm{m}^{2}\right)$ to the next metal level. To investigate possible ways of protection of the MIM capacitor during the processing, various test structures with protection devices have been implemented including the use of diodes and metal bridges. To investigate the antenna perimeter effect, antennas have been designed both as fingers and as plates.

\section{Measurement}

Affected capacitors show a hard breakdown. To detect affected capacitors leakage currents of the test structures are measured at $3.7 \mathrm{~V}\left(V_{\mathrm{dd}+10 \%}\right)$ on the top plate with a grounded bottom plate. The failure criterion is a leakage current $>1 \mu \mathrm{A}$ : fresh capacitors, even the largest capacitors never reach this value, while affected capacitors exceed this value by a few orders of magnitude. The failure fraction is the fraction of failed test structures out of the measured samples. The breakdown voltage of the MIM capacitor dielectric is also investigated. The yield of the product has been measured as well. The experimental vehicle used for this study is a BiCMOS chip with MIM capacitors. The results of the yield test have been split up into the different failure mechanisms of the chip. Wafer maps are provided for each wafer and failure mechanism.

A PDM tool from Semiconductor Diagnostics Inc. is used to detect the process step that causes the charging problem. PDM is a contactless and damage-free measurement to determine the amount of charge built up in an oxide layer [10]. A 1000-nm thermal oxide layer is grown on blank test $\mathrm{Si}$ wafers. Before and after the treatment of the test process step, the potential over the oxide layer can be measured by PDM. The potential difference $\left(\Delta V_{\mathrm{pdm}}\right)$ reflects the charging contribution of this test process step.

\section{RESULTS AND DISCUSSION}

\section{A. $\mathrm{AR}_{(\mathrm{T} / \mathrm{D})}$ and $\mathrm{AR}_{(\mathrm{T} / \mathrm{B})}$}

As previously mentioned, $\mathrm{AR}_{(\mathrm{T} / \mathrm{D})}$ is defined as the area ratio of top antenna versus dielectric of the floating MIM capacitor, and $\mathrm{AR}_{(\mathrm{T} / \mathrm{B})}$ is the area ratio of top antenna versus bottom antenna of the floating MIM capacitor

$$
\begin{aligned}
& A R_{\left(\frac{T}{D}\right)}=\frac{\text { area of Top Antenna }}{\text { area of dielectric of the floating MIM capacitor }} \\
& A R_{\left(\frac{T}{B}\right)}=\frac{\text { area of Top Antenna }}{\text { area of Bottom Antenna }}
\end{aligned}
$$

$\mathrm{AR}_{(\mathrm{T} / \mathrm{D})}$ is commonly used in studying $\mathrm{PCD}$ in the gate oxide of MOS transistors, and the PCD increases with $\mathrm{AR}_{(\mathrm{T} / \mathrm{D})}[11]$. The results presented in Fig. 3, however, show that $\mathrm{AR}_{(\mathrm{T} / \mathrm{B})}$ is a more critical parameter rather than $\mathrm{AR}_{(\mathrm{T} / \mathrm{D})}$ for floating MIM capacitor. In Fig. 3, the failure fraction of different capacitors with the same top antenna is compared. The capacitor is different in size: one has an area of $5 \times 5 \mu \mathrm{m}^{2}$, the other has an area of $20 \times 20 \mu \mathrm{m}^{2}$. A top antenna area of 10720,12400 , or $17200 \mu \mathrm{m}^{2}$ is used. The bottom plate of 


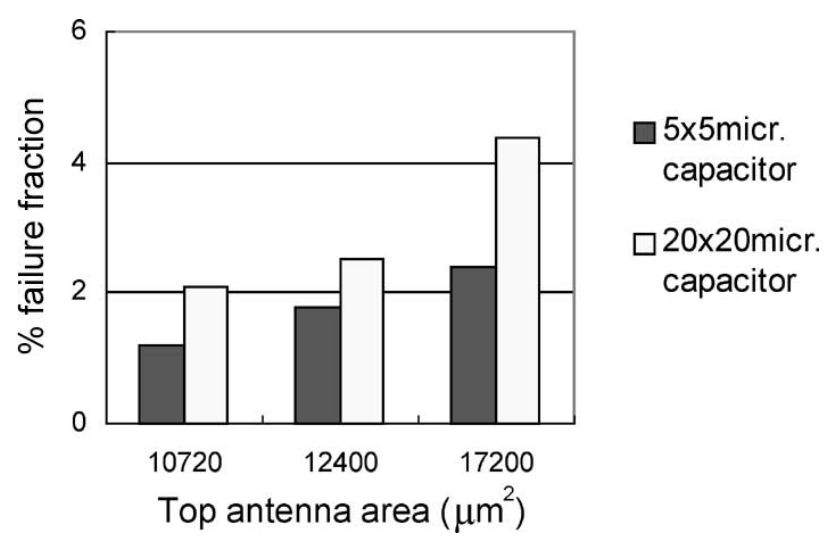

Fig. 3. Failure fraction as a function of the top antenna area for two capacitor sizes. The bottom antenna is $10000 \mu \mathrm{m}^{2}$ in all cases.

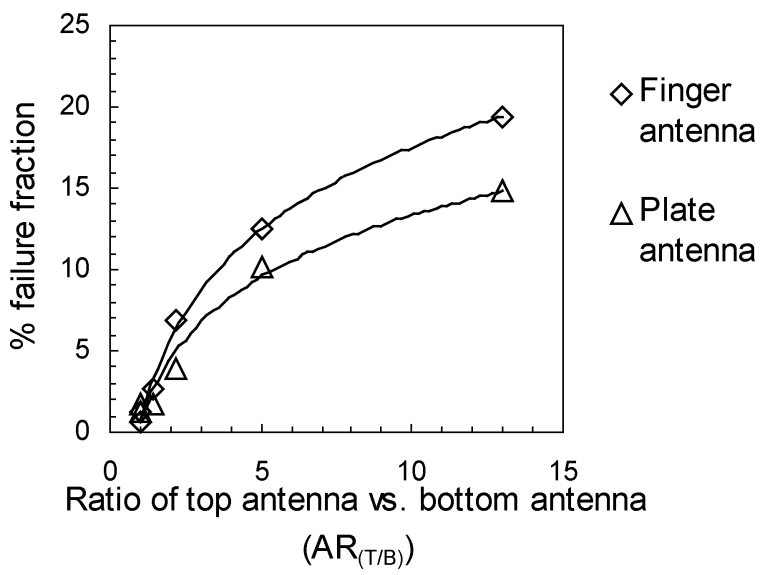

Fig. 4. AR dependence on failure fraction for finger and plate antennas.

all these floating MIM capacitors is connected to a 10000 $\mu \mathrm{m}^{2}$ plate. For the small capacitor, the value of $\mathrm{AR}_{(\mathrm{T} / \mathrm{D})}$ is $16 \times$ higher compared to that of the big capacitor. However, the failure fraction presented in Fig. 3 indicates that, the small capacitor with $16 \times$ higher $\mathrm{AR}_{(\mathrm{T} / \mathrm{D})}$ fails even less than the big capacitor. The two capacitors with the same $\mathrm{AR}_{(\mathrm{T} / \mathrm{B})}$ do not have the same failure fraction. We believe that this can be explained with the role of the capacitor area in the oxide breakdown mechanism. It is well known that, under identical stress conditions, larger area capacitors fail more (or earlier) than small area capacitors [12]. In Section IV, we present a damage model based on $\mathrm{AR}_{(\mathrm{T} / \mathrm{B})}$ rather than $\mathrm{AR}_{(\mathrm{T} / \mathrm{D})}$, which confirms the important role of $A R_{(T / B)}$ in the study of PCD of floating MIM capacitor.

\section{B. Impact of the Antenna Area}

The dependence of $\mathrm{AR}_{(\mathrm{T} / \mathrm{B})}$ on the failure fraction of floating MIM capacitors is shown in Fig. 4. Both top antenna and bottom antenna are on the same metal level and the capacitor is isolated from the substrate. When the top antenna and bottom plate have the same size, the failure fraction falls back to almost zero. The failure fraction increases with an increasing of $\mathrm{AR}_{(\mathrm{T} / \mathrm{B})}$. A potential difference across the insulator between the two plates is generated proportional to the difference in the size of the antennas. When $\mathrm{AR}_{(\mathrm{T} / \mathrm{B})}=1$ (top antenna and bottom plate have the same size), there is no voltage potential difference and as

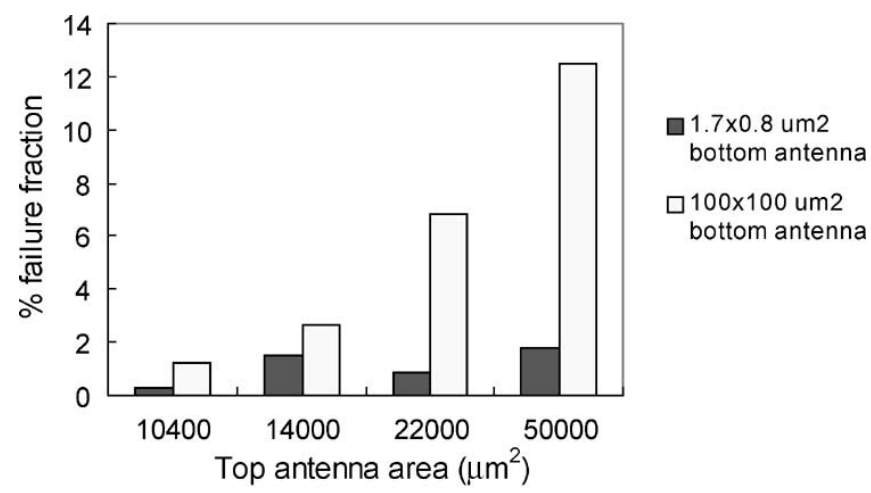

Fig. 5. Effect on PCD of a very small antenna connected to one plate of the floating MIM capacitor.

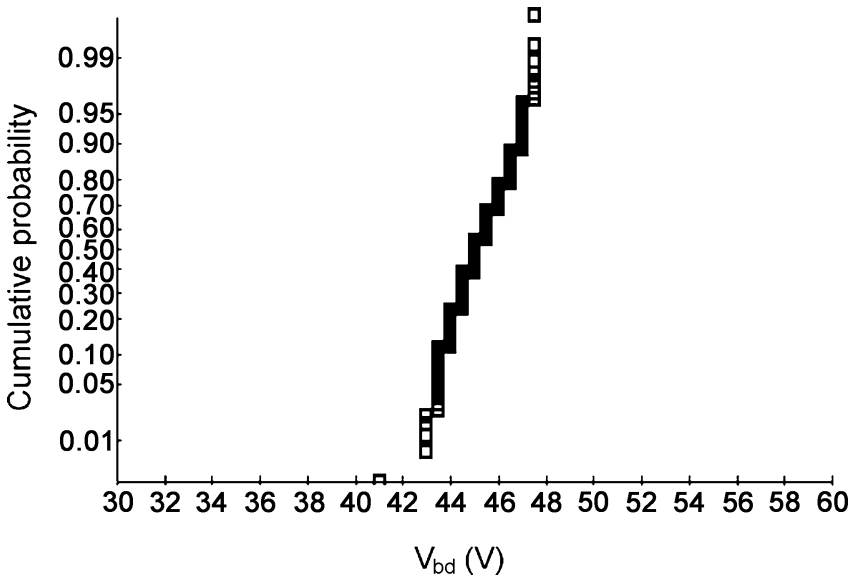

Fig. 6. Breakdown voltage of the dielectric MIM capacitor.

a consequence, there is no PCD. When $\mathrm{AR}_{(\mathrm{T} / \mathrm{B})}$ is large (top antenna is much bigger than bottom antenna), a large voltage potential difference is generated, which causes a high failure fraction.

The bottom plate of some test structures is not connected to an antenna but to an extremely small pad, which is used for connecting to up level. The effects of this very small bottom antenna $\left(1.36 \mu \mathrm{m}^{2}\right)$ versus a large bottom antenna $\left(10000 \mu \mathrm{m}^{2}\right)$ for a range of top antenna sizes are compared in Fig. 5. For the extremely small bottom antenna, the failure fraction remains low and is independent of the top antenna sizes.

\section{Impact of the Antenna Perimeter}

As shown in Fig. 4, the finger antenna structures failed more than plate antenna structures. PCD is more severe with a finger shaped antenna structures compared to a plate-shaped antenna structures with the same antenna area. This must be due to the electron shading effect [13]. Typically, electron shading is very severe during metal etching by plasma tools [14]. The electron shading effect during plasma deposition is also reported in [15], [16].

\section{Source of the Damage}

As shown in Fig. 6, more than $40 \mathrm{~V}$ are required to breakdown a 60-nm-thick dielectric of MIM capacitor. With a PDM tool, it was discovered that the PPRD step in the end of the PECVD process for IMD deposition generated well over $60 \mathrm{~V}$ lateral 


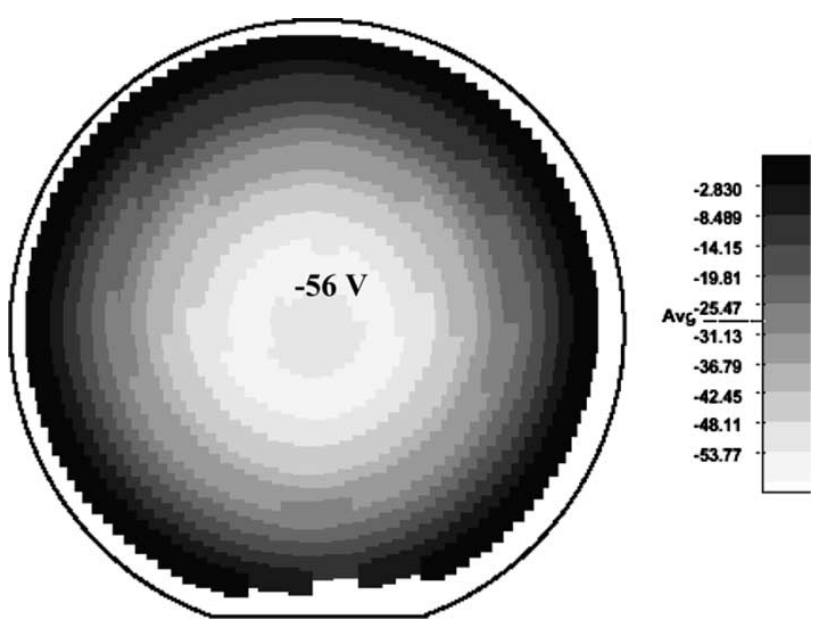

Fig. 7. PDM mapping of a $1000-\AA ̊ \AA$ oxide layer followed by the plasma power ramp down step.

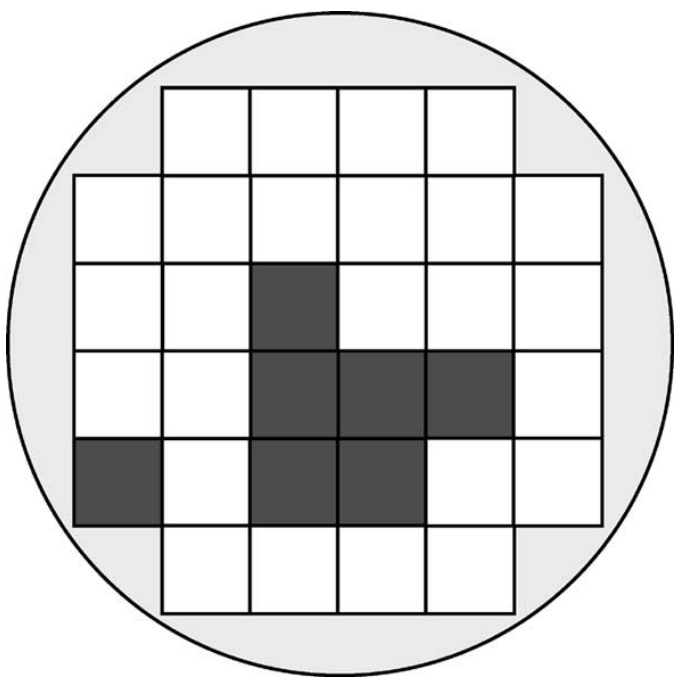

Fig. 8. Typical yield mapping of a wafer processed with the high spin speed rinse process.

potential difference on the surface of the oxide layer between the centre and the edge of the wafer, as observed in Fig. 7. A reduced plasma power helps the reduction of particles. However, it is no longer capable to support a plasma over the full wafer surface: only in the centre of the wafer there is a small region of plasma remaining. This region is charged highly negative as confirmed by the PDM measurement. A voltage of $-56 \mathrm{~V}$ is generated in the centre of the wafer. For the floating MIM capacitors in this highly charged region, the large-area ratio between top antenna and bottom antenna causes a large potential difference across the capacitor dielectric, which causes dielectric breakdown.

The yield mapping result agrees with the PDM result. Fig. 8 illustrate typical mapping of the dysfunctional dices, which fail because of high leakage current. This failure mechanism is strongly related to the leaky MIM capacitors. The dysfunctional dies are mainly located in the region of the centre of the wafer, which coincides with the highly charged region detected by PDM tool. The yield mapping confirms the relation between the charges caused by PPRD step and the failing of MIM capacitors.

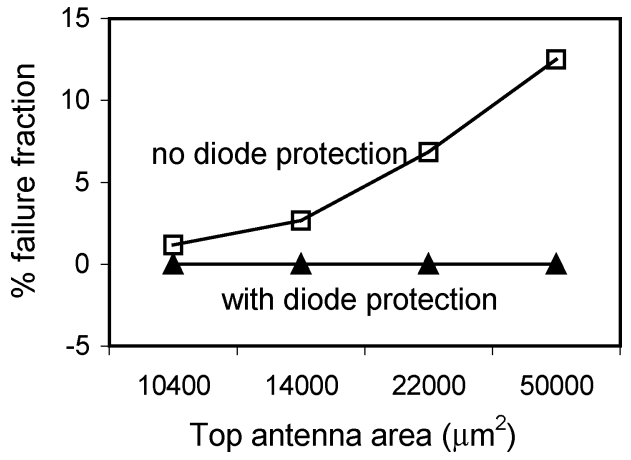

Fig. 9. Effect on PCD of diodes connected to both plates of the floating MIM capacitor.

\section{E. Effect of Diode Protection}

In Fig. 9, the damage to diode protected and unprotected floating MIM capacitors has been compared as a function of the top antenna area. For these test structures, both capacitor plates are connected to $\mathrm{n}+\mathrm{p}$ diode by the same metal level. None of the capacitors protected by diodes fail due to PCD, no matter how large the antennas are. So by using protection diodes, the charges collected on both plates can be discharged efficiently to the substrate through the diodes, limiting potential build-up and preventing PCD to the dielectric. Note that the diodes should be used carefully in order to prevent impact on parasitic capacitance, voltage dependence and quality factors of the MIM capacitors.

\section{DAMAGe Model}

In this section, the model of PCD of floating MIM capacitor is built up. This model agrees with the measurement data of the structures with normal size bottom antenna very well. However, the structure with extremely small bottom antenna is exceptional to this model.

To build up the model to express the relation between the failure fraction of MIM capacitor and $\mathrm{AR}_{(\mathrm{T} / \mathrm{B})}$, well-established dielectric breakdown statistics is used [12]. Through (3) the yield loss (failure fraction) $F$ is linked with the charge $(Q)$ through the oxide [12]

$$
F=1-\exp \left[-\left(\frac{Q}{Q_{63 \%}}\right)^{\beta}\right]
$$

where $\beta$ is the shape parameter or Weibull slope of the distribution and is a known function of the oxide thickness $t_{\mathrm{OX}}$, and $Q_{63 \%}$ is the charge flown through the oxide inducing failure in $1-e^{-1} \approx 63 \%$ of the devices. The dependence of current $(I)$ on $Q_{63 \%}$ can be described by

$$
Q_{63 \%}=K_{0} I^{-K_{1}}
$$

where the parameters $K_{0}$ and $K_{1}$ depend on $t_{\mathrm{OX}}$ and can be defined from a separate experiment.

The charge $Q$ is

$$
Q=I * t
$$


where $t$ is the processing time. By substituting (4) and (5) into (3), the following equation can be obtained

$$
F=1-\exp \left[-\left(\frac{t}{K_{0}}\right)^{\beta} I^{\beta\left(K_{1}+1\right)}\right] .
$$

It was reported before that the damage to MIMC has a logarithmic relation with the AR [17]. The driving force of the damage mechanism is voltage. So now the antenna area dependence of the voltage can be introduced

$$
V=C * \ln \left(A R_{\left(\frac{T}{B}\right)}\right) .
$$

In the low voltage region, nitride dielectric shows an Ohmic conduction mode [18]. Therefore, the current through the floating MIM capacitor can be defined as

$$
I=\frac{V}{R}=C * \frac{\ln \left(\mathrm{AR}_{\left(\frac{T}{B}\right)}\right)}{R}
$$

where AR is the area ratio between the top antenna and bottom antenna of the floating MIM capacitor $\left(\mathrm{AR}_{(\mathrm{T} / \mathrm{B})}\right), R$ is the resistance of the dielectric, and $C$ is a constant. By substituting (8) to (6), the relation between the failure fraction and AR can be obtained

$$
\begin{aligned}
\ln [-\ln (1-F)] & =\beta \ln \left(\frac{t}{K_{0}}\right) \\
& +\beta \ln \left(\frac{C}{R}\right)+\beta\left(K_{1}+1\right) * \ln \left[\ln \left(A R_{\left(\frac{T}{B}\right)}\right)\right] .
\end{aligned}
$$

Defining

$$
D=\beta \ln \left(\frac{t}{K_{0}}\right)+\beta \ln \left(\frac{C}{R}\right)
$$

and

$$
n=\beta\left(K_{1}+1\right) .
$$

Equation (9) can be rewritten as

$$
\ln [-\ln (1-F)]=n * \ln \left[\ln \left(A R_{\left(\frac{T}{B}\right)}\right)\right]+D .
$$

Fig. 10 shows that this function fits the measured data very well, with $n$ equals to 1.03 for finger antennas and 1.04 for plate antennas, and with $D$ equals to 2.48 for finger antennas and 2.83 for plate antennas. We expect that the constants $n$ and $D$ are defined by the antenna shape, the quality of the dielectric, the area of the floating MIM capacitor and the processing itself. Equation (12) allows to anticipate the PCD in the floating MIM capacitors connected to long interconnects, and can be used to define design rules.

There is an exception for the above model, when the bottom antenna is extremely small. As shown in Fig. 10, (9) does not predict the failure fraction which the bottom antenna is limited to a very small area $\left(1.7 \times 0.8 \mu \mathrm{m}^{2}\right)$ which is required to make the connection to a higher metal level. The failure fraction of these extremely small bottom antenna is found to be almost zero even when the other antenna is very large, indicating a different failure mechanism.

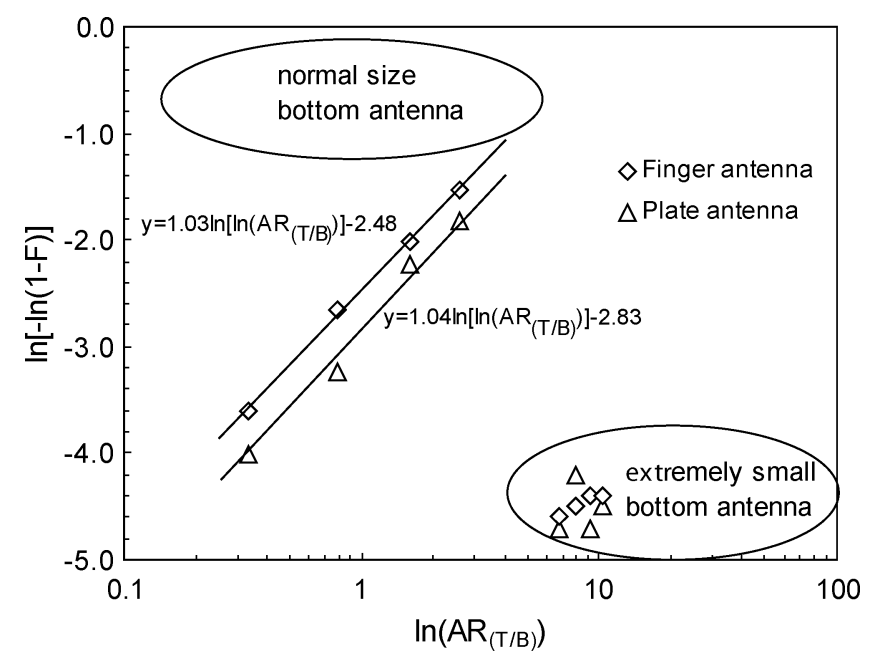

Fig. 10. Simulated and measured failure fraction as a function of the antenna area ratio.

The possible explanation could be that, with a small antenna, only little charge can be exchanged with the environment. As long as the leakage of the floating MIM capacitor dielectric can support this charge transfer, the capacitor will not be damaged. We have only results from devices with an extremely small bottom antenna but we believe this extremely small antenna mechanism is also suitable for a small top antenna. This would imply that if the top antenna is very small, we expect that the floating MIM capacitors will not be damaged no matter how big the bottom antenna is.

\section{LAYOUT SOLUTIONS}

In this section, several layout approaches will be discussed that lead to low plasma damage in line with the model presented above.

\section{A. Using Metal Bridges to Limit Interconnection Line Connected to One of the Two Plates of the Floating MIM Capacitor}

Fig. 5 shows that, for the small bottom antenna, the failure fraction remains low and is independent of the top antenna size on the other plate. This phenomenon is especially interesting because it opens the possibility for the introduction of metal bridges as a way of protecting the floating MIM capacitor. When the ratio of the two conductors connected to the plates of the floating MIM capacitor becomes too large, one of the antennas can be disconnected from the capacitor at the level of the antenna and reconnected again at a higher metal level by means of very small metal area. As such most PCD during the processing is avoided. Fig. 11 shows a possible layout for the protection of the floating MIM capacitors by the use of a metal bridge.

\section{B. Using Protection Diodes to Drain the Charging From the Dielectric of the Floating MIM Capacitor}

Fig. 12 shows a possible layout for the protection of the floating MIM capacitors by the use of $n+/ p$ diodes. Both 


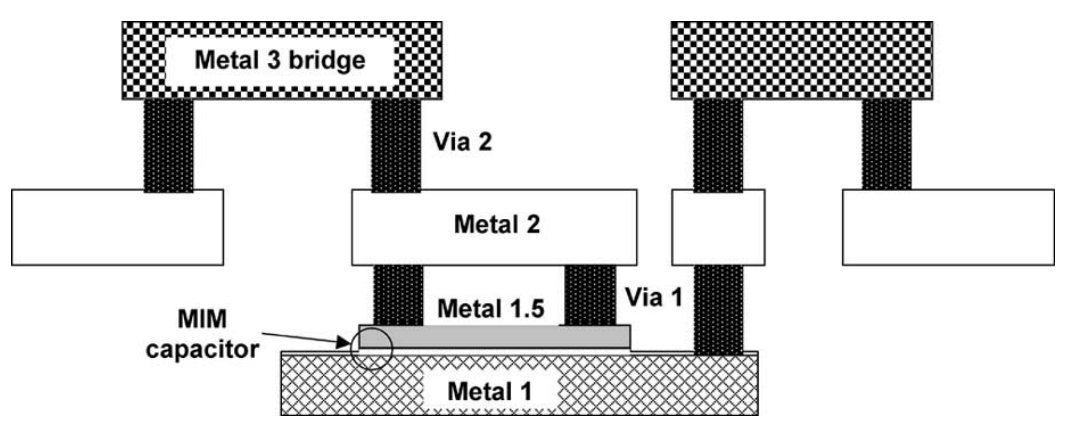

Fig. 11. Possible layout for the protection of the floating MIM capacitors by using metal bridges to limit the connect pad or lines of the bottom plate.

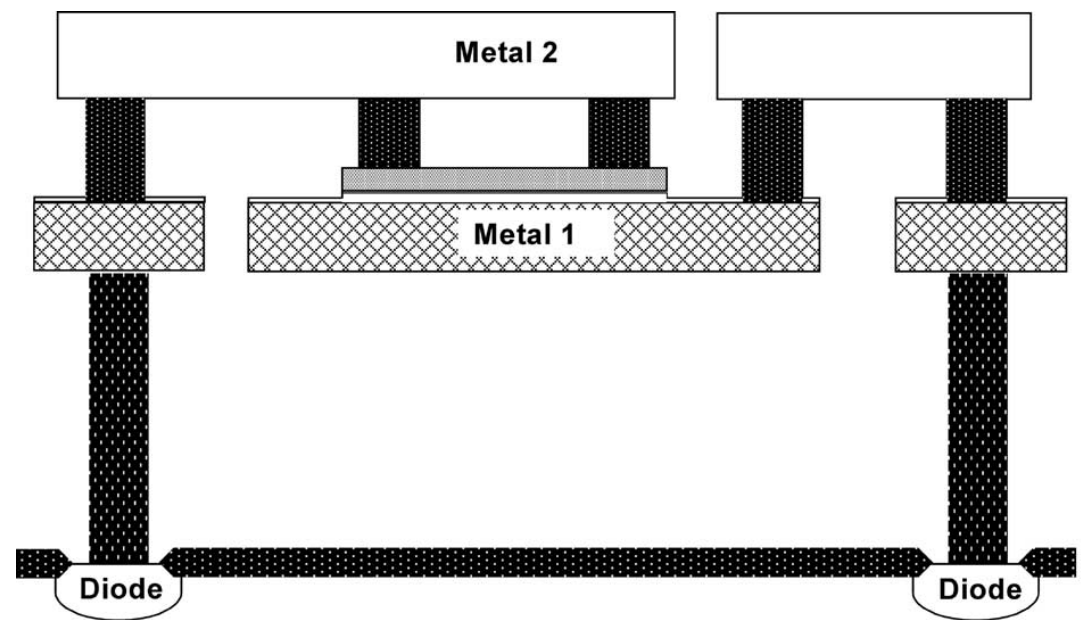

Fig. 12. Possible layout for the protection of the floating MIM capacitors by the use of diodes.

capacitor plates are connected to the diodes by the same metal level. In this way, negative charges collected on both plates can be discharged to the substrate. As such, PCD on the floating MIM capacitor is reduced. The efficacy of the protection diodes has been proven by the experimental results presented in Fig. 9. Special care must be taken that both capacitor plates are connected to the double sided diodes by the same metal level. In the case that only one plate is connected to the substrate by a diode, and the other plate is left floating, all conductors connected to the substrate can act as an antenna of the connected plate. In this case, the ratio between the antenna areas on both plates can be very large. As such, a high a potential difference can be built up across the capacitor, leading to discharge through the capacitor and possibly breakdown.

\section{DESIGN RULES}

The results of the experiments can be summarized in the following simple design rules.

1) Keep the ratio between the areas of the antennas connected to both plates of the floating MIM capacitor for each individual metal level close to unity except for the case that the area of one of the antennas is below a certain threshold value.

2) Contacting only one of the floating MIM capacitor plates to the substrate by a contact, a diode or even by a MOS gate has to be avoided.

3) The use of small metal bridges or protection diodes as proposed in Fig. 11 and Fig. 12 can repair violations of these rules.

\section{CONCLUSION}

In this paper, results from plasma damage experiments on MIM capacitors are presented. The antenna effect is studied and modeled, and design solutions to minimize plasma damage are proposed. PCD to floating MIM capacitors is related to the size and shape of the antennas connected to both capacitor plates. The large area ratio of the interconnects that are connected to the two plates of the capacitor, leads to a potential difference across the insulator between the two plates. This unexpected potential difference stresses the dielectric of the floating MIM capacitor and causes the degradation of the device. Large capacitors are more vulnerable than small capacitors for the same antenna area. Two damage mechanisms describe very well the relation between the failure fraction and the area ratio of antennas connected to the two plates of the floating MIM capacitor. For normal size antennas, the failure fraction is increasing with an increasing difference in top antenna and bottom antenna and falls to almost zero when the top antenna and bottom antenna are identical. For a very small antenna on one plate, the failure fraction remains low and is independent of the antenna size on the other plate. Antenna design rules should be applied during the IC layout phase to prevent the potential PCD during IC manufacturing. By using small metal bridges and protective diodes, the PCD of the floating MIM capacitors can be reduced. The ratio between the areas of the interconnects connected to both plates of the floating MIM capacitor for each individual metal level should be close to unity except for the case that the area of one of the antennas is only a few micrometers. Furthermore, it has to be avoided to contact only one of the floating MIM capacitor plates at a given metal level to the substrate by a contact, a diode or even by a MOS gate. 


\section{REFERENCES}

[1] L. McCreary, "Matching properties and voltage and temperature dependence of MOS capacitors," IEEE J. Solid-State Circuits, vol. SC-16, pp. 608-616, Dec. 1981.

[2] S. P. Tay and J. P. Ellui, "Thin gate and analog capacitor dielectrics for submicron device fabrication," J. Electron. Mater, vol. 21, no. 1, pp. 45-55, 1992.

[3] C. Kaya, H. Tigelaar, J. Paterson, M. de Wit, J. Fattaruso, S. Kiriakai, K. S. Tan, and F. Tsay, "Polycide/metal capacitors for high precision A/D converters," in IEDM Tech. Dig., 1988, pp. 782-785.

[4] A. Yin, J. White, A. Karroy, and C. Hu, "Integration of polycide/metal capacitors in advanced device fabrication," in Proc. 5th Int. Conf. SolidState and Integrated Circuit Technology, 1998, pp. 131-134.

[5] K. Stem, J. Koeis, G. Hueekel, E. Eld, T. Bartush, R. Groves, N. Greco, D. Harame, and T. Tewksbury, "High reliability metal insulator metal capacitors for silicon germanium analog applications," in Proc. BCTM, 1997, pp. 191-194.

[6] S. Decoutere, F. Vleugels, R. Kuhn, R. Loo, M. Caymax, S. Jenei, J. Croon, S. Van Huylenbroeck, M. Da Rold, E. Rosseel, P. Chevalier, an

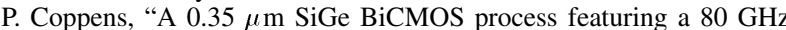
Fmax HBT and integrated high-Q RF passive components," in Proc. BCTM, 2000, pp. 106-109.

[7] S. Fang and J. P. McVittie, "Oxide damage from charging: breakdown mechanism and oxide quality," IEEE Trans. Electron Devices, vol. 41, pp. 1034-1039, June 1994

[8] E. B. Harris, "Charging damage in metal-oxide-metal capacitors," in Proc. 3rd Int. Symp. Plasma Process-Induced Damage (P2ID), 1998, pp. $15-17$

[9] J. Ackaert, Z. Wang, E. Backer, P. Colson, and P. Coppens, "Non contact surface potential measurements for charging reduction during manufacturing of metal-insulator-metal capacitors," Microelectron. Reliab., vol. 41, pp. 1403-1407, 2001

[10] H. M'Saad, S. Desai, D. Witty, C. Hamon, S. Cho, and F. Moghadam, "Plasma-induced defect generation on silicon surfaces in HDP-CVD processing," in Proc. 5th Int. Symp. Plasma Process-Induced Damage $(P 2 I D), 2000$, pp. 42-45.

[11] Z. Wang, A. Scarpa, C. Salm, and F. G. Kuper, "Relation between plasma-process induced oxide failure fraction and antenna ratio," in Proc. 6th Int. Symp. Plasma Process-Induced Damage (P2ID), 2001, pp. 16-19.

[12] R. Degraeve, G. Groeseneken, R. Bellens, J. L. Ogier, M. Depas, P. J. Roussel, and H. E. Maes, "New insights in the relation between electron trap generation and the statistical properties of oxide breakdown," IEEE Trans. Electron Devices, vol. 45, p. 904, Apr. 1998.

[13] K. Hashimoto, "Charge damage caused by electron shading effect," Jpn. J. Appl. Phys., vol. 33, pp. 6013-6018, 1994.

[14] J. Ackaert, "Antenna test structures matrix description, application for optimization HDP oxide deposition, metal etch, Ar preclean and passivation processing in sub-half micron CMOS processing," in Proc. 5th Int. Symp. Plasma Process-Induced Damage (P2ID), 2000, pp. 77-80.

[15] J. P. Carrère, J. C. Oberlin, and M. Haond, "Topographical dependence of charging and new phenomenon during inductively coupled plasma (ICP) CVD process," in Proc. 5th Int. Symp. Plasma Process-Induced Damage (P2ID), 2000, p. 164.

[16] C. T. Gabriel and R. Y. Kim, "Transient fuse structures: The role of metal etching versus dielectric deposition," in Proc. 5th Int. Symp. Plasma Process-Induced Damage (P2ID), 2000, p. 168.

[17] J. Ackaert, Z. Wang, E. Backer, and P. Coppens, "Charging damage in floating metal-insulator-metal capacitors," in Proc. 6th Int. Symp. Plasma Process-Induced Damage (P2ID), 2001, pp. 120-123.

[18] J. Scarpulla, E. D. Ahlers, D. C. Eng, D. L. Leung, S. R. Olson, and C. S Wu, "Dielectric breakdown, defects and reliability in SiN MIMCAPs," in Proc. of GaAs Reliability Workshop, 1998, pp. 92-105.

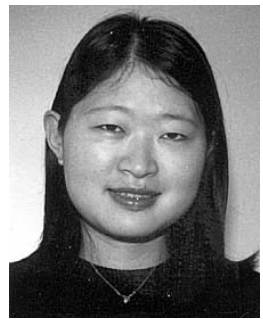

Zhichun Wang (M'99) received the M.Eng. degree in electrical engineering from Xi'an Jiaotong University, Xi' an, China in 1998. She is currently pursuing the Ph.D. degree at the Faculty of Electrical Engineering, University of Twente, Enschede, The Netherlands.

Her research interests include IC technology, semiconductor components reliability and plasma process-induced damage. In 2003, she joined Philips Semiconductors, Nijmegen, The Netherlands, working on front- and backend wafer level reliability.

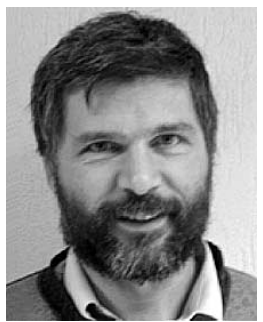

Jan Ackaert was born in Kortrijk, Belgium in 1958. He received the M.S. degree in Chemistry (with honors) from Ostend "Industrial High School (UCB)" in 1980, with a specialization in biochemistry.

After one year with the central laboratory of UCB, he joined Bayer as the Manager of the analytical laboratory and environment. In 1988, he joined AM Semiconductor (former Alcatel Microelectronics), Oudenaarde, Belgium, as Process Support Engineer and later as Process Module Development Engineer for telecom and automotive technologies. His research interests include the effects of the plasma processes on the devices during the manufacturing of the semiconductor. He has published numerous papers on this topic in journals and conference proceedings.

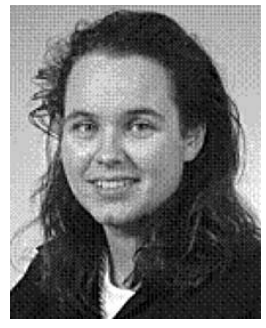

Cora Salm (M'99) was born in Apeldoorn, The Netherlands in 1969. She received the M.Sc. degree in applied physics and the Ph.D. degree in electrical engineering at the University of Twente, Enschede, The Netherlands in 1993 and 1997, respectively. Her Ph.D. dissertation dealt with the deposition, materials, and electrical properties of polycrystalline silicon-germanium.

In 1998, she joined the Semiconductor Components group, MESA+ Research Institute, University of Twente, as an Assistant Professor with a specialization in device reliability. Her research interests include dielectric reliability and failure physics.

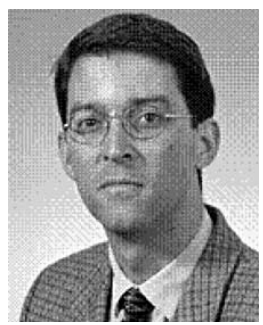

Fred G. Kuper (M'90) received the M.Sc. degree in applied physics from the University of Twente, Enschede, The Netherlands, in 1983. In 1987 he received the Ph.D. degree from the University of Groningen, The Netherlands for his work on stacking faults in silicon.

In 1987, he joined Philips Electronics, working on ESD and front- and backend wafer level reliability. From 1995 to 2002 he headed the reliability group in an 8-in subhalf micrometer CMOS/Flash wafer fab. In 2002, he became Quality Assurance and Reliability Manager of the automotive business line of Philips Semiconductors, Nijmegen, The Netherlands. He is also professor in IC reliability at the MESA+ Research Institute of the University of Twente.

Dr. Kuper serves or has served on Technical Program Committees of IRPS, EOS/ESD Symposium, IEDM, ESREF and INFOS. He received the IRPS 1996 outstanding paper award for his paper on the relation between yield and reliability.

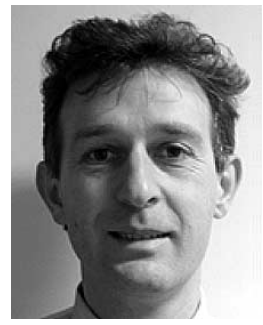

Marnix Tack (M'98) received the M.Sc. degree in electrical engineering from the University of Ghent, Belgium, in 1984, and the Ph.D. degree from the Catholic University of Leuven, Belgium in 1991.

He joined IMEC in 1985 working in the fields of SOI-CMOS and cryogenic CMOS. In 1990, he joined AMI Semiconductor (former Alcatel Microelectronics), Oudenaarde, Belgium, as a Project Leader, later Technology Research and Development Manager, Director and Worldwide Senior Director, being responsible for the development of CMOS, BiCMOS, HV, and NVM processes and design kits. He authored or coauthored over 35 papers and conference contributions in the above fields.

Dr. Tack served as technical program committee member of ESSDERC for several years. 


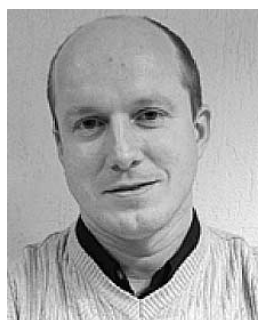

Eddy De Backer (M'98) was born in 1964 in Ghent, Belgium. He received the M.Sc. degree in electrical engineering from the University of Ghent in 1987.

In 1990, he joined AMI Semiconductor, (former Alcatel Microelectronics), Oudenaarde, Belgium as a device integration engineer on the introduction of new CMOS technologies with analogue options. Currently, he is the head of the Process and Module development group. As such, he is responsible for the development of processes and process-modules for all new CMOS, BiCMOS, High Voltage and NonVolatile Memory technology introductions in the AMIS fabs, Oudenaarde.

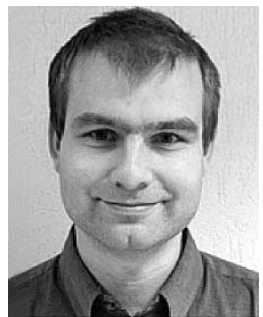

Peter Coppens received the M.Sc. degree in physical engineering from the University of Ghent, Belgium, in 1992.

He joined AMI Semiconductor, (former Alcatel Microelectronics), Oudenaarde, Belgium, in 1994 for the development of CMOS 0.7, 0.5, and 0.35 technologies. From 1998 he is employed as a process module engineer for BiCMOS and HV mixed-signal technologies. For those technologies he developed a high quality metal-insulator-metal capacitor. Currently, he is developing and characterizing deep-trench isolation for the latest high voltage technologies.

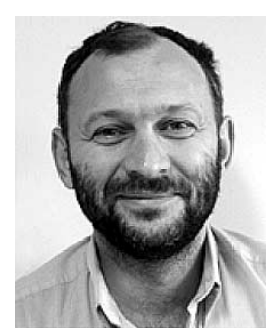

Luc De Schepper was born July, 1962 in Oudenaarde, Belgium. He received the diploma in electronics engineering in 1982 from the PTHTIZottegem, Belgium.

He joined the Technology Research and Development group, AMI Semiconductor, (former Alcatel Microelectronics), Oudenaarde, Belgium, in 1983 as Lab-Coordinator involved in developing and programming of automated measurement setups, measurement methodology, and test benches.

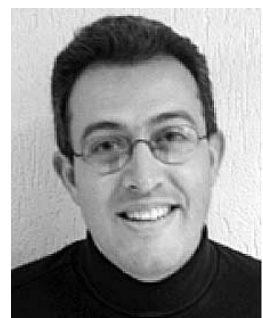

Basil Vlachakis was born in Johannesburg, South Africa, on August 23, 1965. He received the National Higher Diploma Electrical Engineering (Light Current) degree in 1990 at the Technikon Witwatersrand, Johannesburg.

He joined the Technology Research and Development group, AMI Semiconductor, (former Alcatel Microelectronics), Oudenaarde, Belgium, in 1996 as a Laboratory Technician involved in setting up and development of automated measurements, measurement methodology, and test benches. 